\title{
Neutrophil elastase contributes to the pathological vascular permeability characteristic of diabetic retinopathy
}

\author{
Haitao Liu ${ }^{1,2}$ - Emma M. Lessieur ${ }^{3}$ - Aicha Saadane ${ }^{3}$. Sarah I. Lindstrom ${ }^{4}$ Patricia R. Taylor ${ }^{4,5}$ - Timothy S. Kern ${ }^{3,5,6}$
}

Received: 14 April 2019 / Accepted: 31 July 2019/Published online: 14 October 2019

(C) Springer-Verlag GmbH Germany, part of Springer Nature 2019

\begin{abstract}
Aims/hypothesis Levels of neutrophil elastase, a serine protease secreted by neutrophils, are elevated in diabetes. The purpose of this study was to determine whether neutrophil elastase (NE) contributes to the diabetes-induced increase in retinal vascular permeability in mice with streptozotocin-induced diabetes, and, if so, to investigate the potential role of IL-17 in this process. Methods In vivo, diabetes was induced in neutrophil elastase-deficient (Elane ${ }^{-/-}$), Il-17 $a^{-/-}$and wild-type mice. After 8 months of diabetes, Elane ${ }^{-/-}$mice and wild-type age-matched control mice were injected with FITC-BSA. Fluorescence microscopy was used to assess leakage of FITC-BSA from the retinal vasculature into the neural retina. The level of NE in $\mathrm{Il}-17 \mathrm{a}^{-/-}$diabetic retina and sera were determined by ELISA. In vitro, the effect of NE on the permeability and viability of human retinal endothelial cells and the expression of junction proteins and adhesion molecules were studied.

Results Eight months of diabetes resulted in increased retinal vascular permeability and levels of NE in retina and plasma of wildtype animals. All of these abnormalities were significantly inhibited in mice lacking the elastase. The diabetes-induced increase in NE was inhibited in mice lacking IL-17. In vitro, NE increased retinal endothelial cell permeability, which was partially inhibited by a myeloid differentiation primary response 88 (MyD88) inhibitor, NF-KB inhibitor, and protease-activated receptor (PAR)2 inhibitor. NE degraded vascular endothelial-cadherin (VE-cadherin) in a concentration-dependent manner.

Conclusions/interpretation IL-17 regulates NE expression in diabetes. NE contributes to vascular leakage in diabetic retinopathy, partially through activation of MyD88, NF-KB and PAR2 and degradation of VE-cadherin.
\end{abstract}

Keywords Diabetic retinopathy $\cdot$ Elane $\cdot$ IL-17 $\cdot$ Neutrophil elastase $\cdot$ Vascular permeability

Abbreviations

hREC

Human retinal endothelial cell
ICAM-1 Intercellular adhesion molecule 1

INL
Inner nuclear layer
Haitao Liu and Emma M. Lessieur contributed equally to this study.

Electronic supplementary material The online version of this article (https://doi.org/10.1007/s00125-019-04998-4) contains peer reviewed but unedited supplementary material, which is available to authorised users.

Timothy S. Kern

kernt@uci.edu

1 Department of Biology, School of Medicine, Case Western Reserve University, Cleveland, $\mathrm{OH}$, USA

2 Department of Ophthalmology, The First Affiliated Hospital of Dalian Medical University, Dalian, Liaoning 116011, People's Republic of China
3 Center for Translational Vision Research, Department of Ophthalmology, Gavin Herbert Eye Institute, School of Medicine, University of California-Irvine, 829 Health Sciences Rd. Gillespie Neuroscience Research Facility, Room 2107, Irvine, CA 92697, USA

4 Department of Ophthalmology and Visual Sciences, Case Western Reserve University, Cleveland, OH, USA

5 Veterans Administration Medical Center Research Service 151, Cleveland, OH, USA

6 Veterans Administration Medical Center Research Service, Long Beach, CA, USA 


\section{Research in context}

\section{What is already known about this subject?}

- Our laboratory has previously reported that leucocytes play a causal role in the pathogenesis of diabetic retinopathy

- Neutrophil elastase (NE) plays a role in many inflammatory human diseases, including chronic obstructive pulmonary disease, cystic fibrosis, acute lung injury and acute respiratory distress syndrome

- There is an increase in the permeability of the retina in mice with streptozotocin-induced diabetes

\section{What is the key question?}

- Does NE contribute to the diabetes-induced increase in retinal permeability in mice with streptozotocin-induced diabetes, and, if so, is IL-17 also involved?

\section{What are the new findings?}

- $\quad \mathrm{NE}$ contributes to vascular leakage in diabetic retinopathy, partially through activation of MyD88, NF-KB and PAR2 and degradation of a vascular endothelial (VE)-cadherin

- IL-17 regulates NE expression in diabetes

\section{How might this impact on clinical practice in the foreseeable future?}

- The present study shows that a protease released by leucocytes, most probably neutrophils, plays an important role in the development of the diabetes-induced increase in retinal vascular permeability. Thus, NE represents a novel therapeutic target that may potentially inhibit this increase in permeability

IPL

MyD88

NE

ONL

OPL

PAR

SD-OCT

TLR4

VE-cadherin

WT

ZO-1

\section{Introduction}

Diabetic retinopathy is a major complication of diabetes mellitus and an important cause of vision loss among adults of working age [1-4]. Diabetes is known to result in increased permeability of the retinal vasculature, which can contribute to retinal oedema and impaired vision [5]. Additional evidence has demonstrated that retinal endothelial cells and, specifically, the intercellular tight junctions of the retinal endothelial cells, are affected when exposed to the hyperglycaemic conditions of diabetes $[6,7]$. Among the detrimental consequences of exposure to high glucose is the degradation of vascular endothelial cadherin (VE-cadherin), a glycoprotein whose absence leads to increased retinal endothelial cell permeability [8].
Multiple processes have been postulated to account for the retinal vascular lesions observed in diabetic retinopathy. However, the underlying mechanism remains poorly understood. Recent evidence indicates that selective deletion of inflammatory proteins only from marrow-derived cells significantly inhibit retinal capillary degeneration in diabetes [9], and that transgenic expression of neutrophil inhibitory factor (NIF) likewise inhibits the diabetes-induced capillary degeneration $[10,11]$. This evidence suggests that leucocytes play a major role in the development of the early vascular lesions of diabetic retinopathy.

Neutrophil elastase (NE) is a serine protease encoded by the Elane gene (Ensembl-ENSMUST00000046091.6) and is secreted by neutrophils. In addition to its participation in the killing of bacteria and other pathogens, NE has been implicated in diabetes [12] and many inflammatory human diseases, including chronic obstructive pulmonary disease, cystic fibrosis, acute lung injury and acute respiratory distress syndrome [13]. In all these conditions, neutrophils are activated and secrete active NE that contributes to local inflammation and pathology [14-17]. Studies have shown that NE upregulates IL-8 through activation of Toll-like receptor 4 (TLR4) [18, 19]. This upregulation involves the signal-transducing molecules IL-1 receptor-associated kinase (IRAK), myeloid differentiation primary response 88 (MyD88) and TNF-receptorassociated factor 6 (TRAF6). The activation of this pathway, in turn, induces translocation of NF-KB into the nucleus and favours the expression of genes associated with immune and inflammatory responses [19]. In addition, NE has also been 
shown to activate protease-activated receptors (PARs), including PAR2 [20]. PAR2 activation induces NF- $\kappa$ B expression in macrophages [21] and leads to increased transcription of the gene encoding intercellular adhesion molecule-1 (ICAM-1, also known as CD54) in endothelial cells [22, 23].

Whether NE contributes to the vascular leakage observed in diabetes is not known. In the present study, we investigated the possible role of NE in retinal vascular permeability in the early stages of diabetic retinopathy, and a potential mechanism that leads to NE-mediated vascular leakage in a mouse model of diabetes.

\section{Research design and methods}

Ethics statement This study was performed in accordance with the National Institutes of Health Guide for the Care and Use of Laboratory Animals, the Association for Research in Vision and Ophthalmology (ARVO) Statement for the Use of Animals in Ophthalmic and Vision Research, and with the authorisation of the Institutional Animal and Care Use Committee (IACUC) at Case Western Reserve University.

Experimental animals Wild-type (WT) male C57BL/6J, NEdeficient (B6.129X1-Elane tm1Sds $^{\mathrm{J}}$, stock no. 006112) male mice and $\mathrm{Il}-17 \mathrm{a}$-deficient (B6.Cg-Ill $7 \mathrm{ra}^{\mathrm{tm} 2.2 \mathrm{Koll}} / \mathrm{J}$, stock no. 033431) male animals were obtained from the Jackson Laboratory (Ellsworth, ME, USA), with $n>6$ per group. These two strains will be referred to throughout this manuscript as Elane $e^{-/-}$and $I l-17 a^{-/-}$, respectively. All animals were housed in ventilated microisolator cages with free access to water and food. Cages of mice were randomly assigned to be made diabetic, and diabetes was induced at 8-10 weeks of age by intraperitoneal injection of streptozotocin $(60 \mathrm{mg} / \mathrm{kg}$ of body weight) for five consecutive days, as previously described [24]. Mice were assigned numbers upon receipt. $\mathrm{NPH}$ insulin (0-0.2 U) was given subcutaneously to diabetic animals between 0 and 3 times per week to inhibit weight loss, while still allowing hyperglycaemia. Blood glucose was determined with a portable glucose meter (Medline, Northfield, IL, USA), using blood collected from the tail vein under fasting conditions. The onset of diabetes was defined as three consecutive measurements of blood glucose $>15.3 \mathrm{mmol} / 1$. $\mathrm{HbA}_{1 \mathrm{c}}$ was measured every 2-3 months throughout the experiment. In the drug-treatment group, mice who had been diabetic for 5 weeks were treated (i.p. injection) daily for an additional 3 weeks with the NE inhibitor sivelestat (Abcam, Cambridge, MA, USA) at a dosage of $2.0 \mathrm{mg} \mathrm{kg}^{-1}$ day $^{-1}$. Experimenters were masked to group assignment and outcome assessment. No data was excluded from current study.

Optokinetic assessment of photopic visual function The spatial frequency threshold, a marker of visual acuity, and contrast sensitivity threshold were measured in Elane $e^{-/}$and WT mice at 10 months of age ( 8 months' duration of diabetes) with the Virtual Optokinetic system (OptoMotry; CerebralMechanics, Medicine Hat, Canada) as described previously [25]. The maximum spatial frequency capable of driving head tracking was determined as the spatial frequency threshold. The contrast sensitivity was determined at a single point as the inverse of Michelson contrast without correction for luminance of the monitors.

Ultrahigh-resolution spectral-domain optical coherence tomography Ultrahigh-resolution spectral-domain optical coherence tomography (SD-OCT) (Bioptigen, Durham, NC, USA) was used for in vivo imaging of mouse retinas. Elane ${ }^{-/}$and WT mice at 8 months of diabetes (10 months of age) were anaesthetised by intraperitoneal injection of $10 \mu \mathrm{l} / \mathrm{g}$ of diluted ketamine/xylazine $(16.5 / 1.65 \mathrm{mg} / \mathrm{ml})$. Pupils were dilated with $1 \%$ (wt/vol.) tropicamide. Five pictures acquired in the B-scan mode were used to construct each final averaged image. The thickness of the retina and outer nuclear layer (ONL) were measured at distances of 150,300 and $450 \mu \mathrm{m}$ from the optic nerve [26].

Leakage of albumin into neural retina Accumulation of albumin and blood proteins in the neural retina has been regarded as a marker of vascular permeability $[25,27,28]$. At 8 months of diabetes, FITC-BSA (50 $\mu \mathrm{g} / \mu \mathrm{l}$; Sigma-Aldrich; St Louis, $\mathrm{MO}, \mathrm{USA})$ in PBS ( $\mathrm{NaCl}, 0.138 \mathrm{~mol} / \mathrm{l} ; \mathrm{KCl}, 0.0027 \mathrm{~mol} / \mathrm{l}$; $\mathrm{pH}$ 7.4) was injected into the tail vein of mice at $100 \mu \mathrm{g} / \mathrm{g}$ body weight. After $30 \mathrm{~min}$, mice were euthanised and eyes were fixed in $10 \%$ (wt/vol.) formalin for $6 \mathrm{~h}$, followed by incubation in gradient sucrose (up to $20 \%$ [wt/vol.], at least $2 \mathrm{~h}$ each), then frozen in optimal cutting temperature (O.C.T.) compound (Sakura Finetek USA, Torrance, CA, USA). At least four non-contiguous retinal cryosections were cut and imaged by fluorescence microscopy. Leakage of albumin was estimated using computer-assisted microscopy [25, 28] from measurements of FITC-BSA in the inner plexiform layer (IPL), inner nuclear layer (INL) and outer plexiform layer (OPL) of the neural retina and visible blood vessels were excluded. The permeability of the retinas of the diabetic mice was determined as the ratio of FITC-BSA concentration in neural retina relative to that in plasma, and the data were normalised to values in the non-diabetic mice.

Immunohistochemistry Immunofluorescence staining of occludin and zona occludens 1 (ZO-1) was performed as described previously [29]. In brief, retina flat mounts were incubated with the primary antibodies directed against occludin (Thermo Fisher Scientific; Waltham, MA, USA) and ZO-1 (Thermo Fisher Scientific) overnight in 1\% (vol./vol.) Triton X-100 PBS buffer at 1:100 dilution for each antibody. After several washes, Alexa Fluor 488- or 594-conjugated anti- 
mouse or anti-rabbit secondary antibodies (Thermo Fisher Scientific) were applied overnight at $4{ }^{\circ} \mathrm{C}$. Flat mounts were mounted with Prolong Gold anti-fade mounting media (Thermo Fisher Scientific) and analysed on a Leica TCS SP5 confocal microscope (Leica Microsystems, Buffalo Grove, IL, USA) with acousto-optical beam splitter (AOBS).

Human retinal endothelial cell permeability studies To investigate the effect of NE on retinal endothelial cell permeability, Transwell plates $(6.5 \mathrm{~mm}$ insert, $0.4 \mu \mathrm{m}$ polycarbonate membrane; Corning Costar, Kennebunk, ME, USA) were used. Primary human retinal endothelial cells (hRECs) were purchased from Cell Systems (Kirkland, WA, USA). Approximately 10,000 hREC cells $/ \mathrm{cm}^{2}$ were seeded per insert. Cells were grown in the medium for 6 days, and the medium was exchanged every other day. When cells were confluent, FITC-BSA $(250 \mu \mathrm{g} / \mathrm{ml})$ was prepared in the culture medium, the upper compartment culture medium was replaced by $0.2 \mathrm{ml}$ tracer solution, while the lower volume was refreshed with $1 \mathrm{ml}$ culture medium without FITCBSA. For drug treatment, the following reagents were used: human NE (Innovative Research, Novi, MI, USA), sivelestat $(1 \mu \mathrm{mol} / \mathrm{l}$; catalogue no. ab142369, Abcam), TLR4 inhibitor ethyl-(6R)-6-[N-(2-chloro-4-fluorophenyl) sulfamoyl]cyclohex-1-ene-1-carboxylate (catalogue no. tlrlcli95; CLI-095 also known as TAK-242; $3 \mu \mathrm{mol} / 1$; Invivogen, San Diego, CA, USA), MyD88 inhibitor peptide DRQIKIWFQNRRMKWKKRDVLPGT $(100 \mu \mathrm{mol} / 1$; catalogue no. NBP2-29328, Novus Biologicals, Centennial, CO, USA), NF- $\mathrm{kB}$ inhibitor SN50 (18 $\mu \mathrm{mol} / 1$; catalogue no. 2506, BioVision, Milpitas, CA, USA), PAR1 inhibitor 3- $N$ cyclopropyl-7-(4-isopropyl-benzyl)-7H-pyrrolo[3,2f]quinazoline-1,3-diamine dihydrochloride $(1.2 \mu \mathrm{mol} / 1$; catalogue no. Axon 1275, Axon Medchem, Reston, VA, USA), PAR2 inhibitor $N-((S)-3$-cyclohexyl-1- $((2 S, 3 S)-1-(2,3-$ dihydrospiro[indene-1,4'-piperidine]-1'-yl)-3-methyl-1oxopentan-2-ylamino)-1-oxopropan-2-yl)isoxazole-5carboxamide (4 $\mu \mathrm{mol} / 1$; catalogue no. 1622, Axon Medchem), PAR4 inhibitor 1-methyl-5-nitro-3-phenyl-1H-indole-2-methanol (12 $\mu \mathrm{mol} / \mathrm{l}$; catalogue no. 89159-60-4; Cayman Chemical, Ann Arbor, MI, USA). All reagents were added into the medium to the upper chamber as demand. Inserts were taken out after 1, 6 and $12 \mathrm{~h}$ of incubation. FITC fluorescence that had passed through the confluent endothelial cells into the lower chamber was measured using a PerkinElmer 2030 MultiLabel Fluorescence Reader.

Human NE cytotoxicity to hRECs hRECs were set up at a density of 100,000 per well in a six well plate with culture medium with serum and cultureBoost (Cell Systems, Kirkland, WA, USA). The medium was changed every other day for 3 days. When cells reached $90 \%$ of confluence, human NE (Innovative Research, Novi, MI, USA) was added to the
hRECs at concentrations identical to those listed above. Medium (which might contain dead cells) was collected after $12 \mathrm{~h}$, and plates were washed with $2 \mathrm{ml}$ PBS, pH 7.4. Endothelial cells were then removed from the wells by addition of $200 \mu \mathrm{l}$ of $0.05 \%$ (wt/vol.) trypsin for $3 \mathrm{~min}$ and were transferred to $15 \mathrm{ml}$ tubes. Cells then were centrifuged at $200 \mathrm{~g}$ for $8 \mathrm{~min}$, and the pellet re-suspended in $300 \mu \mathrm{PBS}$ (pH 7.4). hREC viability was measured by flow cytometry after immunostaining of endothelial cells with an antibody against CD144 (BD, San Diego, CA, USA) at a dilution of 1:50, and immunostaining of dead cells with 7-AAD (BD). A total of 10,000 events were counted for each sample. Results were analysed using Flow Jo v7.6 Software (FlowJo, Ashland, OR, USA).

Western blot analysis hRECs were seeded at a concentration of 0.5 million per $100 \mathrm{~mm}$ dish, grown for 4 days, and the medium was changed every other day until cells were confluent. Human NE was added to reach final concentrations of 2, 20 and $100 \mathrm{nmol} / \mathrm{l}$. The enzyme was incubated with the cells for $12 \mathrm{~h}$, and cell homogenates were then subjected to western blot analysis. Briefly, extracts of hRECs from different groups were lysed using RIPA buffer and sonication. Lysates were collected by centrifugation at $14,000 \mathrm{~g}$ for $20 \mathrm{~min}$ at $4^{\circ} \mathrm{C}$. Protein concentration was estimated using a Bradford assay. All samples were mixed with Laemmli buffer and boiled for $5 \mathrm{~min}$. Proteins were separated by SDS-PAGE and transferred to nitrocellulose membranes for immunoblotting. Antibodies against VE-cadherin (1:1000 dilution; catalogue no. LSC191761-100, LifeSpan Seattle, WA, USA), ZO-1 (1:1000 dilution; catalogue no. 5406, RRID AB_1904187, Cell Signaling Technology, Dallas, TX, USA), $\beta$-actin (1:1000 dilution; catalogue no. 8457, RRID AB_10950489, Cell Signaling Technology), Occludin, (1:1000 dilution; catalogue no. orb11181, RRID AB_10752986, Biorbyt, San Francisco, CA, USA), claudin-5 (1:1000 dilution; catalogue no. ABT45, RRID AB_11205041, Millipore Sigma, St Louis, MO, USA) and ICAM-1 (1:1000 dilution; catalogue no. 10020-1-AP, RRID AB_2121773, Proteintech, Rosemont, IL, USA) were used. The membrane was incubated with anti-rabbit IgG, HRP-linked secondary antibody (1:1000 dilution; Cell Signaling Technology) in 5\% (wt/vol.) non-fat dry milk at room temperature for $1 \mathrm{~h}$. The membrane was washed in three washes of TBST, $10 \mathrm{~min}$ each. The image was acquired using darkroom development techniques for chemiluminescence.

ELISA The expression of NE in $I l-17^{-/}$mouse retina and sera were measured using an ELISA kit (R\&D Systems, Minneapolis, MN, USA). A total of 5-7 perfused and nonperfused retinas were collected, and pooled for analysis. Serum samples were collected after 2 months of diabetes by lancet cheek blood draw from ten mice. Separate pooled samples were run in triplicate, and each assay was performed at 
least three times. As indicated in kit instructions, samples were prepared at a dilution of 1:1000, and the amount of NE in the test samples was determined by absorbance at $450 \mathrm{~nm}$ using Molecular Devices SpectraMax M5 Multi-Mode Microplate Reader (Boston industries, Walpole, MA, USA), with the amount interpolated from a standard curve constructed from standards after correction for sample dilution.

Statistical analysis Data between groups were analysed by ANOVA followed by Fisher's least significant difference (LSD) post hoc test. When groups were composed of a small number of samples $(n=3)$, the analysis was performed using the Mann-Whitney $U$ test (non-parametric test). A $p$ value of $<0.05$ was considered statistically significant. Data are expressed as mean $\pm \mathrm{SD}$. Results were analyzed using StatView (SAS Institute, Cary, NC, USA).

\section{Results}

Animals Clinical data on non-diabetic and diabetic mice are provided in Table 1 . The severity of diabetes was similar between WT and Elane-deficient mice.

Visual function is not impaired in $E a_{a n e^{-/-}}$mice Spatial frequency threshold and contrast sensitivity were measured in WT and Elane ${ }^{-/}$mice that had been diabetic for 8 months via the optokinetic method. As reported previously, in WT animals, diabetes caused a significant decrease in both measures of visual function (vs non-diabetic WT controls, both $p<0.0001$ ). Neither spatial frequency threshold $(0.398 \pm 0.001,0.311 \pm$ $0.013,0.393 \pm 0.007$ and $0.355 \pm 0.01$ cycle/degree for nondiabetic and diabetic WT and non-diabetic and diabetic Elane $^{-/}$mice, respectively), nor contrast sensitivity (29.1 \pm $3.3,25.9 \pm 3.5,32.2 \pm 2.2$ and $22.3 \pm 3.1$ cycle/degree, respectively) showed any significant effect of NE deficiency on visual function (all $n=5$ per group, data presented as mean $\pm \mathrm{SD}$ ).

The diabetes-induced increase in retinal vascular leakage is inhibited in Elane $^{-/-}$mice Clinical evidence suggests that increased permeability of the retinal vasculature contributes to increased retinal thickness and oedema. We evaluated the permeability of the retinal vasculature at 8 months of diabetes by measuring the amount of FITC-BSA fluorescence that had leaked out of the circulation into the neural retina. The amount of albumin in the neural retina was significantly increased in diabetic WT mice, but this increase was largely abolished in the diabetic Elane $e^{--}$group (Fig. 1). This inhibition of retinal permeability occurred in the IPL (Fig. 1a), INL (Fig. 1b) and OPL (Fig. 1c), each of which contains a vascular plexus of the total retinal vasculature. Representative images from each study group are shown in Fig. 1d. Neither the thickness of the retina (Figs 2a, b) nor the ONL (not shown) was abnormal in any group.

Effects of human NE on permeability across hRECs hRECs were cultured in the insert of Transwell plates for 6 days in order to form tight junctions throughout the culture dish [30]. Human $\mathrm{NE}$ was added to the medium in the insert, and incubated for 1,6 and $12 \mathrm{~h}$, with and without the NE inhibitor sivelestat. Human NE significantly increased endothelial cell permeability in vitro, and this leakage gradually increased in proportion with the incubation time (Fig. 3a). Sivelestat administration was able to completely abolish the effect of NE at $1 \mathrm{~h}$; however, at 6 and $12 \mathrm{~h}$, the inhibitory effect was only partial (although still significant) (Fig. 3a). Administration of sivelestat without NE did not have any effect on retinal endothelial cell permeability.

Given that inflammation can lead to increased cell permeability [31], and the expression of some inflammatory markers is decreased in the absence of NE (T. Kern, unpublished observation), we hypothesised that NE might influence endothelial cell permeability through interaction with inflammatory molecules such as TLR4, MyD88, NF-KB and/or PARs. To test our hypothesis, we determined the increment of permeability in hRECs in the presence of specific inhibitors for the aforementioned inflammatory molecules. We found that the NE-increase in endothelial cell permeability was significantly suppressed by MyD88, NF-kB or PAR2 inhibition, but not by TLR4, PAR1 or PAR4 inhibition (Fig. 3b), suggesting that NE increases endothelial cell permeability via activation of multiple pathways.
Table 1 Clinical data of non-diabetic $(\mathrm{N})$ and diabetic mice (D)

\begin{tabular}{lllcc}
\hline & Group & Body weight $(\mathrm{g})$ & $\mathrm{HbA}_{1 \mathrm{c}}$ & \\
\cline { 4 - 5 } & & & $\%$ & $\mathrm{mmol} / \mathrm{mol}$ \\
\hline Long-term studies (8 months) & WT-N & $49 \pm 5$ & $3.1 \pm 0.1$ & $10 \pm 0.9$ \\
& WT-D & $29 \pm 2 *$ & $11.8 \pm 0.7 *$ & $105 \pm 7.0^{* *}$ \\
& Elane $^{-/-}-\mathrm{N}$ & $40 \pm 4$ & $3.1 \pm 0.1$ & $10 \pm 0.8$ \\
& Elane $^{-/-}$-D & $27 \pm 2 *$ & $11.3 \pm 0.7 *$ & $101 \pm 7.5 * *$ \\
\hline
\end{tabular}

Data are mean $\pm \mathrm{SD}$

$n=5$ for WT-N, $n=6$ per group for others

$* p<0.05,{ }^{*} p<0.01$ compared with non-diabetic (N) from each group

Non-diabetic $(\mathrm{N})$, Diabetic (D) 

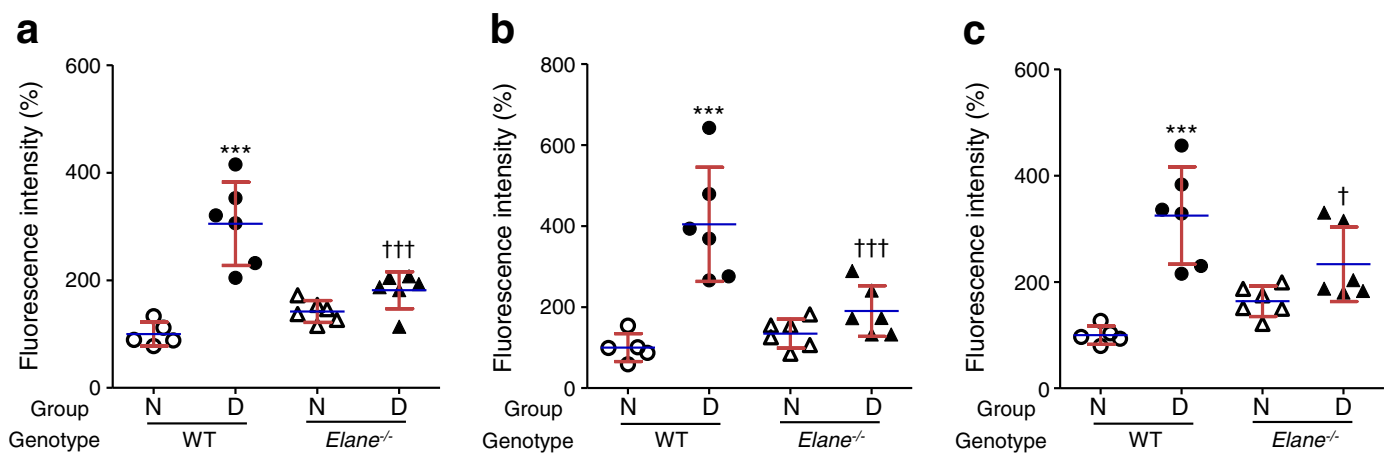

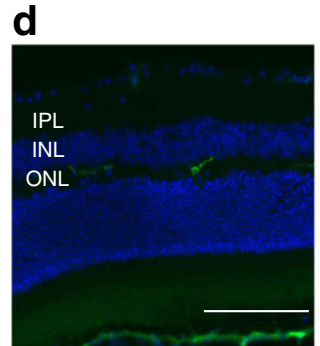

WT-N

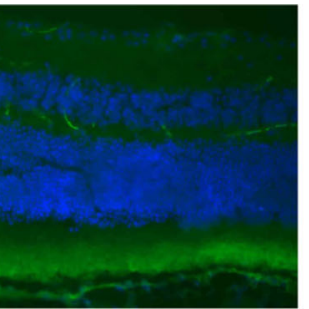

WT-D

Fig. 1 Effects of Elane deletion on vascular permeability in diabetic mice. WT mice with diabetes (WT-D) of 8 months duration significantly increased accumulation of FITC-BSA in the IPL (a), INL (b) and OPL (c) layers of the retina compared with that in WT non-diabetic (WT-N) mice. (d) Representative images from each study group. Genetic deletion of Elane significantly inhibited the extravascular fluorescence in the IPL, INL and OPL of the retina in diabetic Elane ${ }^{-/-}$-D mice. FITC-BSA was

\section{NE-mediated cytotoxicity to endothelial cells is inhibited by} sivelestat in vitro, but not through TLR4, MyD88 or PARs Induction of endothelial cell death might be one contributor to the increase in permeability detected in diabetes. Since we have shown in vitro that NE leads to increased endothelial cell permeability via activation of TLR4, MyD88 and PARs, we investigated whether NE induces endothelial cell death through one or all these pathways. We incubated hRECs with human NE $(50 \mathrm{nmol} / \mathrm{l})$ for $12 \mathrm{~h}$ and found that NE does induce a

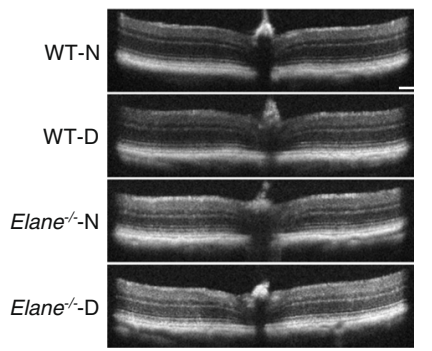

\section{b}

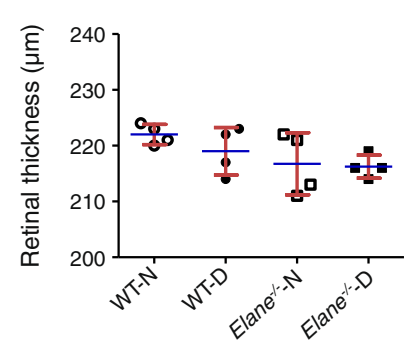

Fig. 2 Effects of Elane deletion and diabetes on retinal thickness. (a) Optical coherence microscopy images of retinas, (b) graph of retinal thickness in experimental groups. Neither diabetes nor Elane ${ }^{-/}$resulted in any significant change in retinal thickness or loss of photoreceptors at 8 months of diabetes (10 months of age). Scale bar, $100 \mu \mathrm{m}, n=4$ per group. Data are expressed as mean $\pm \mathrm{SD}$. Elane ${ }^{-/}-\mathrm{D}$, diabetic Elaneknockout mice; Elane ${ }^{--}-\mathrm{N}$, non-diabetic Elane-knockout mice; WT-D, diabetic WT mice; WT-N, non-diabetic WT mice

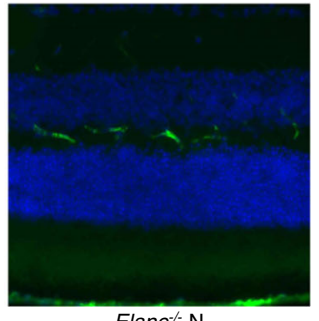

Elane $^{-/-\mathrm{N}}$

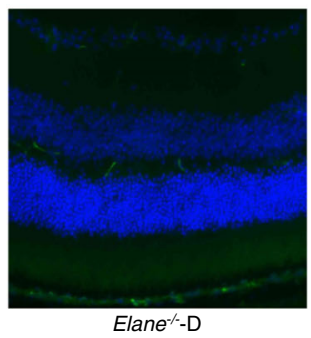

injected intravenously, allowed to circulate for $30 \mathrm{~min}$, and average fluorescence was measured from cross-sections from each layer, excluding microvessels. Data are shown as \% relative to the fluorescence in WT-N mice. Scale bar, $100 \mu \mathrm{m} . n=5$ for WT-N, $n=6$ per group for the others. Data are expressed as mean \pm SD. $* * * p<0.001$ vs WT-N, ${ }^{\dagger} p<0.05,{ }^{\dagger \dagger} p$ $<0.001$ vs WT-D

endothelial cell death, and this was significantly reduced by the addition of sivelestat (Fig. 4). However, in contrast, the NE-induced cytotoxicity in endothelial cells was not inhibited by inhibition of TLR4, MyD88 or PARs, suggesting that endothelial cell death caused by NE activity is not mediated through any of these pathways (Fig. 4).

NE degrades blood-retinal barrier proteins VE-cadherin (also known as CD144) and tight junction proteins are closely related to cell permeability [8]. To determine the mechanism by which human NE increases retinal endothelial cell permeability, we incubated hRECs with NE at different concentrations for $6 \mathrm{~h}$, and evaluated the effect on expression of VE-cadherin, the tight junction proteins ZO-1, occludin and claudin-5, and the adhesion molecule ICAM-1 by immunoblotting (Fig. 5a). NE decreased VE-cadherin expression at a concentration of $20 \mathrm{nmol} / \mathrm{l}$ (Fig. 5b). However, NE did not appear to cleave ZO-1 (Fig. 5c), occludin (Fig. 5d) or claudin-5 (Fig. 5e), indicating that the NEinduced endothelial cell permeability is not due to widespread proteolytic damage to tight junction proteins. Although it has been reported that NE alters expression of ICAM-1, no such effect was seen in our studies (Fig. 5f).

Further investigation of tight junction proteins was conducted in vivo using diabetic animals. Consistent with our in vitro data, the expression of VE-cadherin was decreased significantly in retinas of untreated control diabetic mice, 


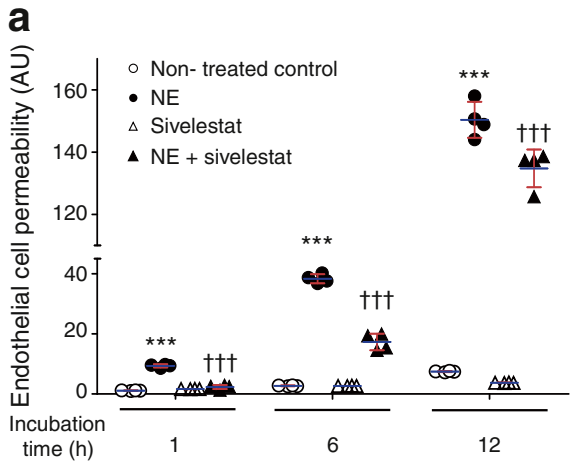

Fig. 3 Effect of human NE on hREC permeability, and possible mediators of this effect. (a) Human NE $(50 \mathrm{nmol} / \mathrm{l})$ directly increased hREC permeability, and this permeability could be inhibited by the NE inhibitor sivelestat. (b) The NE-induced increase in hREC permeability was significantly inhibited by $1 \mathrm{~h}$ incubation with sivelestat; it was significantly inhibited by MyD88 inhibitor peptide, NF- $\mathrm{kB}$ inhibitor and PAR2 inhibitor, but was not inhibited by TLR4 inhibitor, PAR1 inhibitor or PAR4 inhibitor. The complete names and concentrations of inhibitors used are

and the decrease was significantly inhibited in the Elane $e^{--}$ diabetic mice (Figs $5 \mathrm{~g}, \mathrm{~h}$ ). Since a lack of proteolytic cleavage of tight junction proteins in western blots of whole retina does not necessarily mean that there was no cleavage of those proteins in retinal capillaries, preliminary immunofluorescence studies were conducted using retinal whole mounts from nondiabetic and diabetic mice killed at 8 weeks of diabetes. Half of the diabetic mice were treated daily with sivelestat for the 3 weeks prior to autopsy ( $n=2-3$ per group) (electronic supplementary material [ESM] Fig. 1). The data obtained from these retinas suggested that ZO-1 and occludin immunostaining at the junction of vascular endothelial cell membranes were decreased in diabetes, and such abnormality was subtle or not seen in diabetic mice treated with sivelestat (EMS Figs. 1a, b).

Regulation of NE expression by IL-17 Previous studies on vascular permeability in diabetic retinopathy demonstrated that

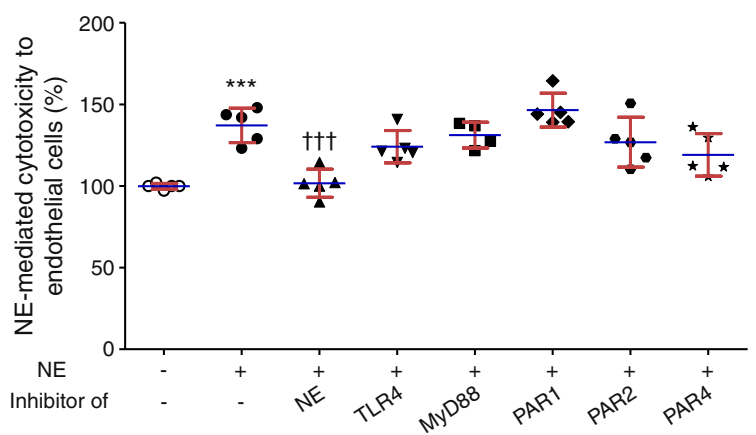

Fig. 4 NE-mediated cytotoxicity to hRECs. Human NE-mediated cytotoxicity towards hRECs was significantly increased after $12 \mathrm{~h}$ incubation compared with non-treated control. Sivelestat significantly inhibited the NE-induced cell death, but inhibition of TLR4, MyD88, PAR1, PAR2 and PAR4 did not inhibit cell death. $n=5$ in duplicate for each group; data are normalised to non-treated control and are expressed as mean \pm SD. $* * * p<0.001$ vs non-treated control, ${ }^{\dagger \dagger} p<0.001$ vs human NE-treated control

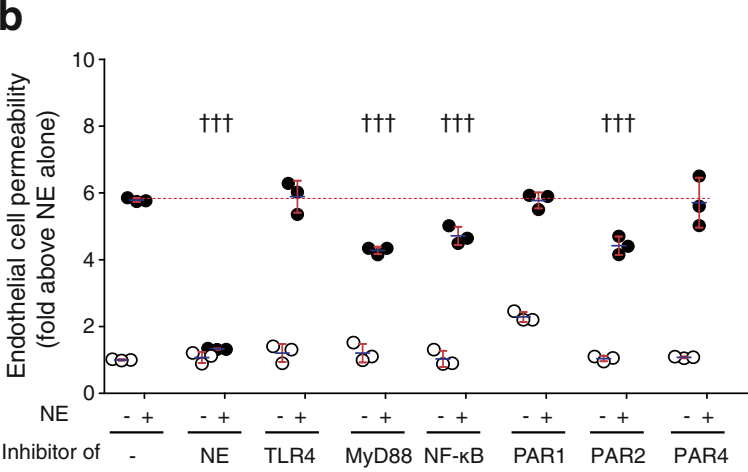

listed in the Methods section. In the figure, replicates incubated with inhibitors but without NE are indicated by white circles, whereas replicates incubated with both the NE and the listed inhibitor are indicated by black circles. $n=4$ replicates for (a), $n=3$ replicates for (b). All samples were measured in duplicate on 2 days (a) or 1 day (b). Data are expressed as mean $\pm \mathrm{SD}$. $* * * p<0.001$ vs non-treated control, ${ }^{\dagger \dagger} p<0.001 \mathrm{vs} \mathrm{hu}-$ man NE-treated control

deletion of IL-17 significantly inhibited the diabetes-induced leakage in the retina [32]. To investigate the relationship between NE expression and IL-17, we measured (by ELISA) NE expression in mice genetically lacking IL-17 [32]. To exclude confounding effects of circulating neutrophils and plasma in the retinal blood vessels on the expression of NE, we studied murine eyes before and after perfusion with saline solution. We found that NE levels were significantly increased in both non-perfused (Fig. 6a) and perfused retinas (Fig. 6b) of WT control diabetic animals, and that expression of NE in serum (Fig. 6c) was also significantly increased in diabetes. In contrast, the levels of NE in retina and serum were significantly lower in diabetic mice lacking IL-17 compared with the levels in diabetic WT animals. Thus, IL-17 plays an important role in the elevation of NE in diabetes, which consequently favours the increase in vascular permeability.

\section{Discussion}

Retinal vascular leakage is a well-established phenomenon that occurs in diabetic retinopathy [25-28, 33, 34]. Abundant clinical evidence provides strong support for an important role of growth factors such as vascular endothelial growth factor (VEGF) in the leakage that happens in advanced stages of retinopathy. However, the genesis of the vascular leakage is less clear. Loss of retinal capillary pericytes in diabetes is known to play a role in the vascular leakage [35], but the demonstrated importance of leucocytes in development of capillary degeneration in early stages of diabetic retinopathy led us to consider the possibility that factors released from such leucocytes also might contribute to the permeability defect. Here, we provide evidence that NE plays an important role in the diabetes-induced increase of retinal vascular 
a
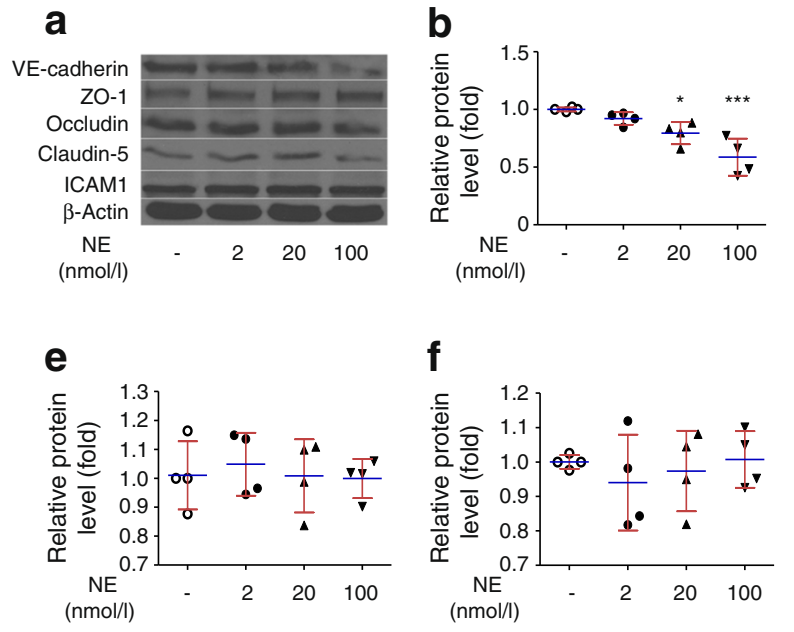

Fig. 5 Effects of NE on the expression of VE-cadherin, the junction proteins ZO-1, occludin, and claudin-5, and ICAM-1. (a) Representative blots of effects of $12 \mathrm{~h}$ exposure of hRECs to various concentrations $(2 \mathrm{nmol} / \mathrm{l}, 20 \mathrm{nmol} / \mathrm{l}$ and $100 \mathrm{nmol} / \mathrm{l})$ of human NE on proteins. (b) VE-cadherin was degraded by human NE in a concentrationdependent manner. The expression of ZO-1 (c), occludin (d), claudin-5 (e) and ICAM-1 (f) did not seem to be affected by human NE. (g, h)

permeability that develops in the early stages of diabetic retinopathy. Diabetes of 8 months duration in WT mice led to accumulation of fluorescently labelled albumin in the neural retina, whereas the diabetes-induced permeability defect was significantly inhibited in animals lacking NE. However, this degree of permeability, was not sufficient to cause retinal oedema. In general, no studies of diabetic rodents have reported thickening of the retina due to leakage, with the exception of one report in diabetic rats [36]. The inhibition of the diabetesinduced increase in retinal vascular permeability shown in our NE-deficient mice is significant but is not total. Undoubtedly, there are other factors that also contribute to the permeability defect, including matrix metalloproteinases (MMPs), as reported previously [8].

NE in the circulation is known to be continuously exposed to an inhibitor of its proteolytic activity ( $\alpha_{1}$ antitrypsin), and
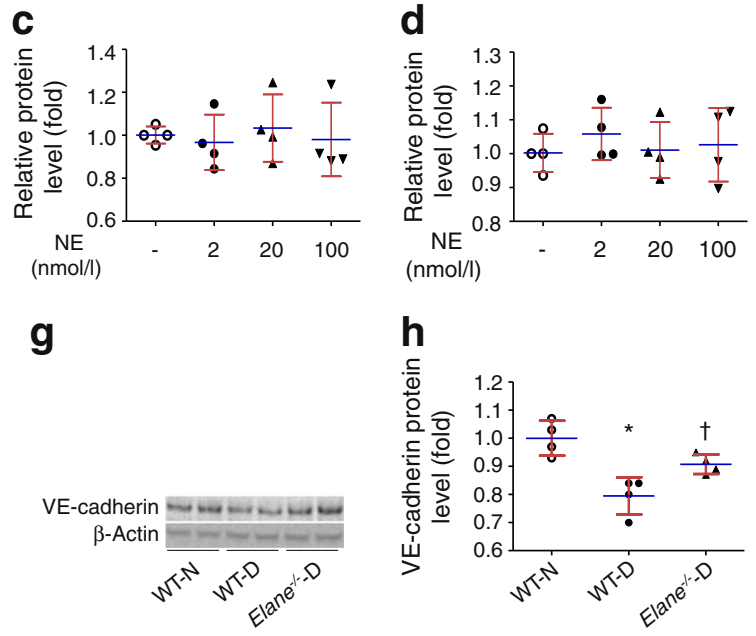

Immunoblot from mouse retina showing decreased expression of VEcadherin in diabetic animals vs non-diabetic control animals. Elane ${ }^{-1-}$ D, diabetic Elane-knockout mice. WT-D, diabetic WT mice; WT-N, nondiabetic WT mice Decreased expression of VE-cadherin was partially corrected in diabetic mice lacking NE. $* p<0.05$, *** $p<0.001$ vs nontreated control or WT-N, ${ }^{\dagger} p<0.05$ vs WT-D. $n=4$ replicates for $(\mathbf{a}-\mathbf{f})$. $n=4$ mice for each group in (g) and (h). Data are expressed as mean \pm SD

so it is reasonable to question how NE could cause any adverse effects in diabetes. It has been reported that NE bound to the cell surface [37], especially in the cleft that forms between leucocytes adhered to endothelial cells, is resistant to inhibition by naturally occurring proteinase inhibitors [38]. In addition, non-enzymatic glycation in diabetes has been reported to reduce the protease inhibitory activity of $\alpha_{1}$ antitrypsin [39].

Studies have shown that chemokine (C-X-C motif) ligand 8 (CXCL8) is upregulated in human bronchial epithelial cells through activation of TLR4 by purified NE, followed by activation of MyD88 and NF- $\mathrm{KB}$ [19]. Also, activation of PARs by $\mathrm{NE}$ is reported to activate NF-KB, resulting in increased transcription of ICAM-1 [22, 23]. In our in vitro study, endothelial cell permeability was significantly increased by incubation with purified human NE, and this increase was partially blocked by inhibitors of MyD88, NF-KB and PAR2, but not

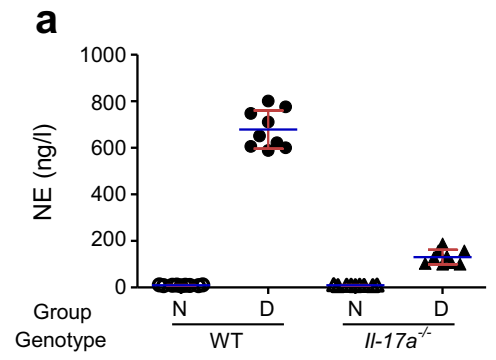

Fig. 6 Expression of NE in $\mathrm{Il}-17 a^{-/-}$mouse retina and sera. Diabetes significantly increased the expression of $\mathrm{NE}$ in the non-perfused retina (a), perfused retina (b), and sera (c). They are all significantly decreased in $\mathrm{Il}-17 a^{-/}$mice. The protective effects on retinal vascular permeability from $I l-17 a^{-/}$mice [44] may be followed by a reduction of NE. For the perfused retina, each sample was 7 retinas pooled; 3 separate pooled b

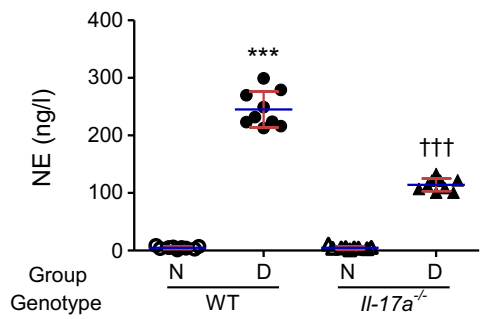

samples were run in triplicate. For the non-perfused retina, each sample was 5 retinas pooled; 3 separate pooled samples were run in triplicate. Each serum sample was pooled from lancet cheek draw from ten mice, drawn 2 months after they became diabetic; 3 separate pooled samples were run in triplicate. Data are expressed as mean \pm SD. $* * * p<0.001$ vs WT-N, ${ }^{\dagger \dagger} p<0.001$ vs WT-D 
by $1 \mathrm{~h}$ incubation with inhibitors of TLR4, PAR1 and PAR4. These data indicate that the NE-induced increase in endothelial cell permeability may be mediated via complex inflammatory signalling pathways. Endothelial cell death also contributes to the increased retinal vasculature permeability in diabetes [33, 40]. Our data are consistent with this idea, since we found that purified NE causes cytotoxicity against endothelial cells. However, we found that the cell death was not inhibited by any of the inhibitors listed above, indicating that NE increased cell permeability through PAR2, MyD88 and NF-KB is independent of endothelial cytotoxicity. How NE causes endothelial cell death requires further investigation.

VE-cadherin and thee junction proteins ZO-1, occludin and claudin-5 are closely associated with vascular permeability in diabetes [6-8], and the proteolytic activity of NE could contribute to the reduction in levels of these proteins $[13,41]$. NE cleaves epithelial cadherin in acutely injured lung epithelium, and increases epithelial permeability in a time- and concentration-dependent fashion $[42,43]$. In line with results from other investigators [8], in this study we found that purified human NE degrades VE-cadherin in a dose-dependent manner. Furthermore, preliminary studies suggested detrimental effects of NE on expression of other barrier proteins of the retinal vasculature. That the expression of ICAM-1 in the retinal vasculature is increased by diabetes has been established for years, but some publications also reported that expression of ICAM-1 was altered by NE [44]. In our study, we did not see any evidence of NE-induced changes in ICAM-1 expression in endothelial cells. Our results show, however, that genetic deletion of IL-17 significantly inhibited the diabetesinduced increase in levels of NE in the retina and serum. These data suggest that the diabetes-induced retinal vascular leakage may be mediated, at least in part, via IL-17 regulation of NE and its activity.

In summary, we provide evidence that $\mathrm{NE}$ contributes to the vascular leakage in the early evolution of diabetic retinopathy, potentially via PAR2, MyD88 and NF-kB signalling. These data further emphasise the role of leucocytes and inflammatory processes in the development of diabetic retinopathy and suggest that protease inhibition may represent a novel therapeutic target to inhibit important components of the pathways involved in the genesis of retinopathy.

Acknowledgements The authors thank D. A. Antonetti and X. Liu (University of Michigan, Ann Arbor, MI, USA) for the retinal whole mount immunostaining. Chieh Allen Lee, Katie Franke and Heather Butler (Case Western Reserve University, Cleveland, OH, USA) who maintained the mouse colonies. Dawn Smith (Case Western Reserve University) who maintained the hRECs.

Data availability The datasets generated during and/or analysed during the current study are available from the corresponding author on reasonable request. Other data pertaining to other abnormalities from the longterm study will be published separately, but all data pertaining to permeability in this study are included in this published article (and its ESM).

Funding This work was supported by NIH grants RO1 EY022938 and R24 EY024864 (to TSK), and grants BX003604 (to TSK) and BX003403 (to PRT) from the Department of Veterans Affairs, and core grant P30 EY011373 to Case Western Reserve University. 81900884 (to HL) from The First Affiliated Hospital of Dalian Medical University. This research received no specific grant from any funding agency in commercial or notfor-profit sectors.

Duality of interest The authors declare that there is no duality of interest associated with this manuscript.

Contribution statement HL performed molecular analyses and wrote the manuscript. EML analysed data and wrote the manuscript. AS and PRT acquired data, were involved in the analysis and interpretation of data and reviewed the manuscript. SIL performed ELISA, was involved in the analysis and interpretation of data and edited the manuscript. TSK designed experiments, acquired data and reviewed/edited the manuscript. All the authors approved the final version of the manuscript to be published. TSK is the guarantor of this work.

\section{References}

1. Lee R, Wong TY, Sabanayagam C (2015) Epidemiology of diabetic retinopathy, diabetic macular edema and related vision loss. Eye Vis 2(1):17. https://doi.org/10.1186/s40662-015-0026-2

2. Ogurtsova K, da Rocha Fernandes JD, Huang Y et al (2017) IDF Diabetes Atlas: global estimates for the prevalence of diabetes for 2015 and 2040. Diabetes Res Clin Pract 128:40-50. https://doi.org/ 10.1016/j.diabres.2017.03.024

3. Solomon SD, Chew E, Duh EJ et al (2017) Diabetic retinopathy: a position statement by the American Diabetes Association. Diabetes Care 40(3):412-418. https://doi.org/10.2337/dc16-2641

4. Engelgau MM, Geiss LS, Saaddine JB et al (2004) The evolving diabetes burden in the United States. Ann Intern Med 140(11):945950. https://doi.org/10.7326/0003-4819-140-11-200406010-00035

5. Klaassen I, Van Noorden CJ, Schlingemann RO (2013) Molecular basis of the inner blood-retinal barrier and its breakdown in diabetic macular edema and other pathological conditions. Prog Retin Eye Res 34:19-48. https://doi.org/10.1016/j.preteyeres.2013.02.001

6. Joussen AM, Smyth N, Niessen C (2007) Pathophysiology of diabetic macular edema. Dev Ophthalmol 39:1-12

7. Tien T, Barrette KF, Chronopoulos A, Roy S (2013) Effects of high glucose-induced $\mathrm{Cx} 43$ downregulation on occludin and $\mathrm{ZO}-1$ expression and tight junction barrier function in retinal endothelial cells. Invest Ophthalmol Vis Sci 54(10):6518-6525. https://doi.org/10. 1167/iovs.13-11763

8. Navaratna D, McGuire PG, Menicucci G, Das A (2007) Proteolytic degradation of VE-cadherin alters the blood-retinal barrier in diabetes. Diabetes 56(9):2380-2387. https://doi.org/10.2337/db06-1694

9. Li G, Veenstra AA, Talahalli RR et al (2012) Marrow-derived cells regulate the development of early diabetic retinopathy and tactile allodynia in mice. Diabetes 61(12):3294-3303. https://doi.org/10. 2337/db11-1249

10. Joussen AM, Poulaki V, Le ML et al (2004) A central role for inflammation in the pathogenesis of diabetic retinopathy. FASEB J 18(12):1450-1452. https://doi.org/10.1096/fj.03-1476fje

11. Veenstra AA, Tang J, Kern TS (2013) Antagonism of CD11b with neutrophil inhibitory factor (NIF) inhibits vascular lesions in diabetic retinopathy. PLoS One 8(10):e78405. https://doi.org/10.1371/ journal.pone.0078405 
12. Wang Y, Xiao Y, Zhong L et al (2014) Increased neutrophil elastase and proteinase 3 and augmented NETosis are closely associated with beta-cell autoimmunity in patients with type 1 diabetes. Diabetes 63(12):4239-4248. https://doi.org/10.2337/db14-0480

13. Korkmaz B, Horwitz MS, Jenne DE, Gauthier F (2010) Neutrophil elastase, proteinase 3 , and cathepsin $\mathrm{G}$ as therapeutic targets in human diseases. Pharmacol Rev 62(4):726-759. https://doi.org/ $10.1124 /$ pr. 110.002733

14. Lee WL, Downey GP (2001) Leukocyte elastase: physiological functions and role in acute lung injury. Am J Respir Crit Care Med 164(5):896-904. https://doi.org/10.1164/ajrccm.164.5. 2103040

15. Moraes TJ, Chow CW, Downey GP (2003) Proteases and lung injury. Crit Care Med 31(Suppl):S189-S194. https://doi.org/10. 1097/01.CCM.0000057842.90746.1E

16. Owen CA (2008) Roles for proteinases in the pathogenesis of chronic obstructive pulmonary disease. Int J Chron Obstruct Pulmon Dis 3:253-268. https://doi.org/10.2147/COPD.S2089

17. Shapiro SD (2002) Proteinases in chronic obstructive pulmonary disease. Biochem Soc Trans 30(2):98-102. https://doi.org/10.1042/ bst0300098

18. Walsh DE, Greene CM, Carroll TP et al (2001) Interleukin-8 upregulation by neutrophil elastase is mediated by MyD88/IRAK/ TRAF-6 in human bronchial epithelium. J Biol Chem 276(38): 35494-35499. https://doi.org/10.1074/jbc.M103543200

19. Devaney JM, Greene CM, Taggart CC, Carroll TP, O’Neill SJ, McElvaney NG (2003) Neutrophil elastase up-regulates interleukin-8 via toll-like receptor 4. FEBS Lett 544(1-3):129-132. https://doi.org/10.1016/S0014-5793(03)00482-4

20. Soh UJ, Dores MR, Chen B, Trejo J (2010) Signal transduction by protease-activated receptors. Br J Pharmacol 160(2):191-203. https://doi.org/10.1111/j.1476-5381.2010.00705.x

21. Ritchie E, Saka M, Mackenzie C et al (2007) Cytokine upregulation of proteinase-activated-receptors 2 and 4 expression mediated by p38 MAP kinase and inhibitory kappa B kinase beta in human endothelial cells. Br J Pharmacol 150(8):1044-1054. https://doi. org/10.1038/sj.bjp.0707150

22. Hung DT, Wong YH, Vu TK, Coughlin SR (1992) The cloned platelet thrombin receptor couples to at least two distinct effectors to stimulate phosphoinositide hydrolysis and inhibit adenylyl cyclase. J Biol Chem 267(29):20831-20834

23. Rahman A, True AL, Anwar KN, Ye RD, Voyno-Yasenetskaya TA, Malik AB (2002) $G \alpha_{q}$ and $G \beta \gamma$ regulate PAR-1 signaling of thrombin-induced NF-KB activation and ICAM-1 transcription in endothelial cells. Circ Res 91(5):398-405. https://doi.org/10.1161/ 01.RES.0000033520.95242.A2

24. Veenstra A, Liu H, Lee CA, Du Y, Tang J, Kern TS (2015) Diabetic retinopathy: retina-specific methods for maintenance of diabetic rodents and evaluation of vascular histopathology and molecular abnormalities. Curr Protoc Mouse Biol 5(3):247-270. https://doi. org/10.1002/9780470942390.mo140190

25. Liu H, Tang J, Du Y et al (2015) Retinylamine benefits early diabetic retinopathy in mice. J Biol Chem 290(35):21568-21579. https://doi.org/10.1074/jbc.M115.655555

26. Liu H, Tang J, Du Y et al (2016) Photoreceptor cells influence retinal vascular degeneration in mouse models of retinal degeneration and diabetes. Invest Ophthalmol Vis Sci 57(10):4272-4281. https://doi.org/10.1167/iovs.16-19415

27. Antonetti DA, Barber AJ, Khin S, Lieth E, Tarbell JM, Gardner TW (1998) Vascular permeability in experimental diabetes is associated with reduced endothelial occludin content: vascular endothelial growth factor decreases occludin in retinal endothelial cells. Penn State Retina Research Group. Diabetes 47(12):1953-1959. https:// doi.org/10.2337/diabetes.47.12.1953

28. Du Y, Cramer M, Lee CA et al (2015) Adrenergic and serotonin receptors affect retinal superoxide generation in diabetic mice: relationship to capillary degeneration and permeability. FASEB J 29(5):2194-2204. https://doi.org/10.1096/fj.14-269431

29. Muthusamy A, Lin CM, Shanmugam S, Lindner HM, Abcouwer SF, Antonetti DA (2014) Ischemia-reperfusion injury induces occludin phosphorylation/ubiquitination and retinal vascular permeability in a VEGFR-2-dependent manner. J Cereb Blood Flow Metab 34(3):522-531. https://doi.org/10.1038/jcbfm.2013.230

30. Nooteboom A, Hendriks T, Otteholler I, van der Linden CJ (2000) Permeability characteristics of human endothelial monolayers seeded on different extracellular matrix proteins. Mediat Inflamm 9(5): 235-241. https://doi.org/10.1080/09629350020025755

31. Tonade D, Liu H, Palczewski K, Kern TS (2017) Photoreceptor cells produce inflammatory products that contribute to retinal vascular permeability in a mouse model of diabetes. Diabetologia 60(10):2111-2120. https://doi.org/10.1007/s00125-017-4381-5

32. Sigurdardottir S, Zapadka TE, Lindstrom SI, Liu H, Lee CA, Kern TS, Taylor PR (2019) Diabetes-mediated IL-17A enhances retinal inflammation, oxidative stress, and vascular permeability. Cell Immunol 341: 103921. https://doi.org/10.1016/j.cellimm.2019.04.009

33. Frey T, Antonetti DA (2011) Alterations to the blood-retinal barrier in diabetes: cytokines and reactive oxygen species. Antioxid Redox Signal 15(5):1271-1284. https://doi.org/10.1089/ars.2011.3906

34. Vinores SA, Derevjanik NL, Mahlow J, Berkowitz BA, Wilson CA (1998) Electron microscopic evidence for the mechanism of bloodretinal barrier breakdown in diabetic rabbits: comparison with magnetic resonance imaging. Pathol Res Pract 194(7):497-505. https:// doi.org/10.1016/S0344-0338(98)80118-0

35. Beltramo E, Porta M (2013) Pericyte loss in diabetic retinopathy: mechanisms and consequences. Curr Med Chem 20(26):3218 3225. https://doi.org/10.2174/09298673113209990022

36. Berkowitz BA, Bissig D, Ye Y, Valsadia P, Kern TS, Roberts R (2012) Evidence for diffuse central retinal edema in vivo in diabetic male Sprague Dawley rats. PLoS One 7(1):e29619. https://doi.org/ 10.1371/journal.pone.0029619

37. Owen CA, Campbell MA, Sannes PL, Boukedes SS, Campbell EJ (1995) Cell surface-bound elastase and cathepsin G on human neutrophils: a novel, non-oxidative mechanism by which neutrophils focus and preserve catalytic activity of serine proteinases. J Cell Biol 131(3):775-789. https://doi.org/10.1083/jcb.131.3.775

38. Yu X, Akbarzadeh R, Pieper M et al (2018) Neutrophil adhesion is a prerequisite for antibody-mediated proteolytic tissue damage in experimental models of epidermolysis bullosa acquisita. J Investig Dermatol 138(9):1990-1998. https://doi.org/10.1016/j.jid.2018.03.1499

39. Hashemi M, Naderi M, Rashidi H, Ghavami S (2007) Impaired activity of serum alpha-1-antitrypsin in diabetes mellitus. Diabetes Res Clin Pract 75(2):246-248. https://doi.org/10.1016/j.diabres.2006.06.020

40. Joussen AM, Doehmen S, Le ML et al (2009) TNF- $\alpha$ mediated apoptosis plays an important role in the development of early diabetic retinopathy and long-term histopathological alterations. Mol Vis $15: 1418-1428$

41. Mecham RP, Broekelmann TJ, Fliszar CJ, Shapiro SD, Welgus HG, Senior RM (1997) Elastin degradation by matrix metalloproteinases. Cleavage site specificity and mechanisms of elastolysis. J Biol Chem 272(29):18071-18076. https://doi.org/10.1074/jbc.272.29.18071

42. Boxio R, Wartelle J, Nawrocki-Raby B et al (2016) Neutrophil elastase cleaves epithelial cadherin in acutely injured lung epithelium. Respir Res 17(1):129. https://doi.org/10.1186/s12931-016-0449-x

43. Peterson MW, Walter ME, Nygaard SD (1995) Effect of neutrophil mediators on epithelial permeability. Am J Respir Cell Mol Biol 13(6):719-727. https://doi.org/10.1165/ajrcmb.13.6.7576710

Publisher's note Springer Nature remains neutral with regard to jurisdictional claims in published maps and institutional affiliations. 